\title{
Valoración de la herramienta Dolceta: una herramienta de aprendizaje electrónico para primaria y secundaria
}

\author{
Maria Victòria Sánchez-Rebull, Fernando Campa-Planas, \\ Ana Beatriz Hernández Lara \\ Universidad Rovira i Virgili \\ mariavictoria.sanchez@urv.cat; fernando.campa@urv.cat; \\ anabeatriz.hernandez@urv.cat
}

Resumen: El objeto del presente trabajo es describir la valoración efectuada sobre Dolceta, una herramienta en línea para la alfabetización financiera de alumnos de primaria, secundaria y bachillerato. Tras realizar un grupo de discusión, se han llevado a cabo once entrevistas a profesores de secundaria conocedores de esta herramienta. Los resultados del análisis de estas entrevistas y del grupo de discusión ponen de manifiesto el interés mostrado por este recurso virtual, que es visto, a su vez, como un medio y como una oportunidad, tanto para emplear herramientas audiovisuales en linea en el aula que permiten el learning by doing, como para aportar una cultura financiera a los estudiantes que en la situación actual de crisis financiera se percibe como un activo más que necesario. Asimismo, se demuestra también su utilidad para los adultos. El presente trabajo aporta opiniones profesionales sobre la aplicabilidad práctica de una herramienta de formación en línea. Al tratarse de una plataforma de aprendizaje electrónico de libre acceso, se evitan restricciones de tipo económico o espacial, y todos los ciudadanos pueden acceder a ella libremente. Como conclusión, se constata la posibilidad real de utilizar el módulo de "Alfabetización financiera» de la plataforma Dolceta, especialmente, en un entorno educativo de primaria y secundaria, tanto en España como en el resto de estados de la Unión Europea.

Palabras clave: Educación del consumidor, alfabetización financiera y aprendizaje electrónico. 
Dolceta assessment tool: an e-learning tool for primary and secondary studies Abstract: The purpose of this study is to describe the assessment of Dolceta, an online tool for financial learning of students in primary and secondary school. After conducting a focus group, interviews were developed with 11 high school teachers as potential users of Dolceta. The results of the analysis of these interviews and focus group show their interest in this virtual resource that is seen both as a tool and as an opportunity. They consider that the on-line tool in the classroom allow «learning by doing", and provide financial knowledge to students that, in the current financial crisis situation, is seen as an asset more than necessary. Also, it is pointed out its usefulness to adulthood people. The present study provides professional opinions on the practical applicability of a tool of on-line training. Being an e-learning platform under free access conditions, all citizens have free access without considering geographical or economics restrictions. As a conclusion, a real possibility to use the financial module Dolceta platform exists, especially in a primary and secondary education, both in Spain and in the rest of the European Union.

Keywords: Consumer education, financial education and e-learning. 


\section{Introducción}

La utilización de las tecnologías de la información y la comunicación (TIC) presenta posibilidades y retos de innovación que pueden comportar un cambio en el proceso de enseñanza-aprendizaje y una mejora de la calidad docente. A su vez, ofrecen oportunidades a los alumnos para perfeccionar algunas competencias de cara a su futura vida laboral (Ho y Dzeng, 2010).

El espectacular desarrollo de las TIC, especialmente de internet, está transformando radicalmente la enseñanza y el aprendizaje (Ma, Vogel y Wagner, 2000). La enseñanza en línea permite que más personas puedan acceder a la educación. Se les ofrecen nuevas formas de aprender y estudiar, lo que provoca la aparición de nuevas herramientas, materiales, actividades en línea y procesos que facilitan el aprendizaje y la interacción entre profesores y alumnos sin restricción de tiempo o espacio (Barker, 2002; Burgess, 2007; Benito, 2009; Cabero y Gisbert, 2005; y Sun y otros, 2008), y se les proporciona un entorno en el que desarrollar un aprendizaje más autónomo para convertirse en constructores activos de su propia formación, sin ser solo meros receptores pasivos (Fu, Su y Yu, 2009). Estos cambios transforman las TIC en un nuevo paradigma de la educación moderna (Sun y otros, 2008), por lo que estas y el aprendizaje basado en entornos web han emergido recientemente como líneas de investigación relevantes (McGill y Klobas, 2009).

Este desarrollo se ha concretado en el uso de medios audiovisuales, la informática y las tecnologías avanzadas, la creación de entornos virtuales educativos (por ejemplo, Moodle o Alexia), las herramientas de simulación, etc., que han tenido gran aceptación y resultados notables en todos los niveles educativos.

En este contexto, Dolceta (Development of Online Consumer Education Tool for Adults) es un proyecto educativo en línea impulsado por la Unión Europea. Implica a 28 países europeos y pretende desarrollar la educación para el consumo basándose en las TIC. Está promovido por la Dirección General de Sanidad y Consumo (DG SANCO), y ha sido desarrollado técnicamente por la European University Continuing Education Network (EUCEN). Ofrece módulos en línea para los consumidores, con información útil para tomar decisiones seguras de compra y sobre temas vinculados al consumo, como los derechos de los consumidores, los servicios, la seguridad de los productos, el consumo sostenible, los servicios financieros o la alfabetización financiera.

Precisamente, este trabajo, de carácter descriptivo, pretende analizar la percepción de potenciales usuarios de la herramienta Dolceta, en concreto del módulo «Alfabetización financiera», como recurso de aprendizaje electrónico, destinado especialmente a edades tempranas (alumnos de primaria, secundaria y bachille- 
rato). Así, se quiere contribuir a la escasa literatura sobre aprendizaje electrónico existente en el ámbito financiero a través de la descripción de la valoración realizada respecto a una herramienta de formación en línea correspondiente a un proyecto de educación europeo y poniendo de manifiesto la importancia de promover la alfabetización financiera para apoyar decisiones económico-financieras cotidianas.

\section{Objetivo del módulo "Alfabetización financiera» de Dolceta}

El propósito del módulo «Alfabetización financiera» es apoyar a los profesores de primaria, secundaria y bachillerato ofreciéndoles las herramientas necesarias para desarrollar y ejecutar programas educativos relacionados con instrumentos y problemas financieros actuales, y facilitar información y recursos para la ciudadanía en el ámbito de la alfabetización financiera. Esta alfabetización debe ser homogénea en los países europeos implicados en el proyecto y adaptarse al contexto de cada país. Esta necesidad se satisface mediante las TIC en esta plataforma educativa.

Este objetivo es importante dada la creciente necesidad de educación de los consumidores a escala europea (Bailey y Kitson, 2006; y Declaración de Bolonia, 1999), lo que puede extrapolarse a otros ámbitos, incluso al internacional. Los consumidores exigen más que nunca una formación explícita que les ayude a gestionar situaciones cotidianas de consumo complejas (Carnell y Lodge, 2002; y Stoll, Fink y Earl, 2004) ante un contexto actual, caracterizado por profundos cambios: la globalización, la crisis financiera, la introducción de las TIC y los productos financieros complejos. Por ello, se hace evidente la necesidad de disponer de habilidades genéricas y específicas (Declaración de Bolonia, 1999; y Ministerio de Educación, Cultura y Deporte, 2003), así como de conocimientos técnicos que ayuden a enfrentarse exitosamente a decisiones de consumo diarias.

No obstante, sorprende que la literatura previa al respecto sea escasa. La mayor parte trata de describir experiencias específicas de enseñanza en línea o casos de aplicación de alguna herramienta de aprendizaje electrónico sobre finanzas o consumo, como simuladores de bolsa, juegos de negocios, etc. (Robinson, 2008; y Yamashita, Takahashi y Terano, 2008).

En los últimos años, a raíz de la grave crisis económica, han aparecido trabajos que han hecho hincapié en la necesidad de difundir información financiera y de consumo en la sociedad (Arvanitis, 2010). Las características de los productos financieros se han revelado más complejas que las de otros productos (Arvanitis, 2010), y la información disponible sobre estos es escasa para el usuario final y poco comprensible para los no profesionales. Los expertos reunidos en el Se- 
gundo Encuentro Europeo sobre Educación Financiera concluyeron que, aunque existen limitaciones específicas, la crisis financiera supone una oportunidad para promover la educación financiera en la Unión Europea y facilitarla a los ciudadanos (Comisión Europea, 2009). Existen algunas iniciativas previas en educación financiera entre los estados miembros (Habschick, Seidl y Evers, 2007), aunque con escaso intercambio de estas experiencias entre ellos.

En el caso de los productos financieros, los consumidores deben afrontar el desafío de la alfabetización financiera como una experiencia de formación continua (Alrø y Skovsmose, 2000; y Bailey y Kitson, 2006). Por ello, es importante dotar a los profesores de las herramientas necesarias para fomentar una alfabetización financiera de calidad desde edades tempranas.

\section{Estructura del módulo «Alfabetización financiera»}

El módulo incluye una introducción — que responde a las siguientes preguntas: ¿Por qué enseñar alfabetización financiera?, ¿cómo enseñarla? y ¿qué enseñar?-, cuatro secciones, un glosario de términos financieros y fichas técnicas especializadas. Cada sección se desarrolla en tres niveles por edades (hasta 10 años, 15 y 18). Partiendo de una propuesta desarrollada por un panel de expertos europeos, cada sección está dividida en seis lecciones (tabla 1) (Sánchez, Campa y Hernández, 2011).

Tabla 1. Lecciones del módulo para la plataforma española.

\begin{tabular}{|c|c|c|c|c|}
\hline & \multicolumn{4}{|c|}{ Secciones } \\
\hline Nivel & Gastos & $\begin{array}{l}\text { Ahorro } \\
\text { e inversión }\end{array}$ & $\begin{array}{l}\text { Préstamos } \\
\text { y créditos }\end{array}$ & $\begin{array}{c}\text { Seguros } \\
\text { y protección }\end{array}$ \\
\hline \multirow[t]{2}{*}{ Primaria } & $\begin{array}{l}\text { Organizar una } \\
\text { fiesta }\end{array}$ & $\begin{array}{l}\text { Invertir mis } \\
\text { ahorros }\end{array}$ & $\begin{array}{c}\text { Tarjetas de crédito } \\
\text { y de débito }\end{array}$ & $\begin{array}{c}\text { ¿Estamos seguros? } \\
\text { ¿Qué podemos } \\
\text { hacer para estarlo? }\end{array}$ \\
\hline & $\begin{array}{l}\text { El dinero y su } \\
\text { historia }\end{array}$ & $\begin{array}{l}\text { ¿Qué puedo hacer } \\
\text { yo para ahorrar? }\end{array}$ & Compras a plazos & Tipos de seguros \\
\hline \multirow[t]{2}{*}{ Secundaria } & $\begin{array}{c}\text { Divisas y moneda } \\
\text { extranjera }\end{array}$ & Banca ética & Microcrédito & $\begin{array}{l}\text { Cuando las cosas } \\
\text { van mal, ¿cómo } \\
\text { ponemos una } \\
\text { denuncia? }\end{array}$ \\
\hline & De compras & $\begin{array}{l}\text { Calcular mis } \\
\text { ahorros }\end{array}$ & $\begin{array}{c}\text { Intereses y } \\
\text { vencimiento de un } \\
\text { préstamo }\end{array}$ & $\begin{array}{l}\text { ¿Qué hacemos si } \\
\text { una compra no nos } \\
\text { ha salido bien? }\end{array}$ \\
\hline \multirow{2}{*}{ Bachillerato } & $\begin{array}{l}\text { Planificar un } \\
\text { viaje }\end{array}$ & Abrir un negocio & $\begin{array}{l}\text { Préstamos a } \\
\text { estudiantes }\end{array}$ & Principales seguros \\
\hline & $\begin{array}{l}\text { Aprender a hacer } \\
\text { un presupuesto }\end{array}$ & La bolsa & $\begin{array}{l}\text { Comprar una casa } \\
\text { con una hipoteca }\end{array}$ & $\begin{array}{c}\text { ¿Podemos } \\
\text { cuantificar el riesgo? }\end{array}$ \\
\hline
\end{tabular}

Fuente: Elaboración propia 


\section{Metodología aplicada}

La evaluación de la calidad de la herramienta la llevan a cabo normalmente los usuarios o los potenciales usuarios, como el profesor (Ozkan y Koseler, 2009) o el estudiante (Teo y Wong, 2013; y Chen y otros, 2011). Este estudio se centra, precisamente, en la evaluación desde la óptica del profesor.

Se utilizaron dos metodologías. Primero, se desarrolló un grupo de discusión con once integrantes, dos entrevistadores y dos personas de apoyo para pilotar la entrevista y recoger sugerencias con el fin de mejorarla. Segundo, se realizaron nueve entrevistas a personas distintas a las del grupo de discusión, a profesionales dedicados a la formación de alumnos de bachillerato y de ESO.

Tabla 2. Resumen de las características de los entrevistados.

\begin{tabular}{|c|c|c|c|c|}
\hline & \multicolumn{2}{|c|}{ Titulación universitaria } & Máster & $\begin{array}{c}\text { Años de } \\
\text { experiencia }\end{array}$ \\
\hline 1 & \multicolumn{2}{|c|}{ Derecho } & $\begin{array}{l}\text { Formación del Profesorado } \\
\text { (Administración de Empresas) }\end{array}$ & 2 \\
\hline 2 & \multicolumn{2}{|c|}{ Publicidad } & Formación del Profesorado & 2 \\
\hline 3 & \multicolumn{2}{|c|}{$\mathrm{ADE}$} & Formación del Profesorado & 15 \\
\hline 4 & \multicolumn{2}{|c|}{ Ciencias Económicas } & Formación del Profesorado & 11 \\
\hline 5 & \multicolumn{2}{|c|}{ Derecho } & Formación del Profesorado & 14 \\
\hline 6 & \multicolumn{2}{|c|}{ Arquitectura Técnica } & $\begin{array}{l}\text { Posgrado de Urbanismo y máster de } \\
\text { Formación del Profesorado }\end{array}$ & 8 \\
\hline 7 & $\begin{array}{l}\text { Ciencia } \\
\text { Política }\end{array}$ & Periodismo & Formación del Profesorado & 7 \\
\hline 8 & \multicolumn{2}{|c|}{ Ciencias Empresariales } & Formación del Profesorado & 4 \\
\hline 9 & \multicolumn{2}{|c|}{ Ciencias Empresariales } & Formación del Profesorado & 7 \\
\hline
\end{tabular}

Fuente: Elaboración propia

La etapa incipiente de este recurso en línea no facilita que todos los entrevistados utilicen Dolceta, aunque están valorando su aplicación. No obstante, su perfil (tabla 2) se considera adecuado, pues todos están capacitados para impartir temas financieros en diferentes niveles educativos. 


\section{Resultados obtenidos}

A continuación, se describirán los resultados obtenidos de las entrevistas.

\subsection{Ventajas e inconvenientes del aprendizaje electrónico}

Es frecuente la literatura referida a las ventajas y los inconvenientes del aprendizaje electrónico y de las herramientas en línea (experiencias positivas y negativas en los procesos de aprendizaje electrónico) desde la óptica de los usuarios (Chen, Lin y Kinshuk, 2008; Fitó-Bertrán, Hernández-Lara y Serradell-López, 2014 y 2015; y Hernández, Gorjup y Cascón, 2010).

Los entrevistados destacaron las siguientes ventajas del aprendizaje electrónico:

- Mejora de las habilidades relacionadas con las TIC sobre la movilidad por internet: «Estás enseñando el mismo contenido que puedes enseñar en un papel, pero enseñándoles también a moverse ellos mismos por una red, a buscar» (entrevistado 1 ).

- Contribución a la realización de un mayor esfuerzo: «Está muy bien para aprender, y aquel que quiera, aprende, e incluso más que, quizás, con un libro y con una clase magistral, porque te esfuerzas más» (entrevistado 1).

- Facilidad de acceso fuera del aula: «Lo puedes preparar en casa, no necesitas estar en el aula» (entrevistado 8).

Se percibe positivamente la existencia de diferentes recursos de aprendizaje electrónico como complemento para preparar las clases, por ejemplo: «Son herramientas muy útiles para dar clase, porque es otro recurso que puedes usar... como apoyo, en ocasiones, incluso mejoran el contenido» (entrevistado 9) y «[Es] interesante para completar el trabajo del docente» (entrevistado 6).

Como inconvenientes, los entrevistados manifestaron los siguientes puntos:

+ Dificultad para motivar a los estudiantes a aprender a utilizar nuevas herramientas: «El trabajo es motivar a los estudiantes para que aprendan a usarlas y [se familiaricen con ellas]» (entrevistado 6).

+ Necesidad de ser constanteः «En el aula aprendes más [... que si no eres una persona constante, a distancia» (entrevistado 8).

- Necesidad de refuerzo presencial: «Es útil siempre que se refuerce con un examen presencial» (entrevistado 5). Así pues, se percibe como una herramienta que debe combinarse necesariamente con una explicación teórica por parte del profesor en clase, o bien como recurso complementario al trabajo realizado en el aula.

+ Dificultad para comprobar la autoría del trabajo del estudiante. 


\subsection{Conocimiento previo sobre herramientas de aprendizaje electrónico}

A pesar de las bondades del aprendizaje electrónico, los entrevistados manifiestan conocer pocas herramientas; sobre todo, saben de la existencia de campus virtuales (Universitat Oberta de Catalunya o Moodle): «Sí, campus virtuales, plataformas, vaya» (entrevistado 2).

Nadie conocía el módulo objeto de estudio, aunque se había desarrollado años atrás. Conocían otras herramientas en línea que publican materiales y actividades, aunque sin feedback de los errores cometidos al resolverlas, y según los entrevistados, menos estructuradas que Dolceta. Planteaban la fiabilidad de la fuente como aspecto importante:

Sí, conozco herramientas, más específicas... el glosario del Banco de España, cosas básicas como Wikipedia y otras más especializadas de información financiera, pero no tan orientadas [...] al mundo educativo (entrevistado 6).

\subsection{Posibilidades de utilización del módulo «Alfabetización financiera»}

La mayoría de los entrevistados consideran factible utilizar este módulo en clase, especialmente en bachillerato y secundaria, porque los alumnos suelen disponer de ordenadores. De no tenerlos, proponen usarlo en el aula de informática, aunque algún entrevistado indica que puede aplicarse en clase con y sin ordenadores.

Se baraja la idea de utilizarlo puntualmente en clase para cambiar el ritmo, cuando los alumnos estén cansados. Este resultado encaja con la literatura, que señala la diversión como una característica que las herramientas de aprendizaje electrónico aportan al proceso educativo (Fu, Su y Yu, 2009).

Este módulo se concibe como un recurso útil para temas cotidianos de los ciudadanos, que pueden beneficiarse de este y otros módulos de Dolceta: «También tienes todos los recursos para poder aprender toda la lección tú en tu casa» (entrevistado 1).

Concretamente, en la situación financiera actual, se considera útil, aunque debería haber estado disponible antes. En España, en la actualidad, el inicio de la enseñanza de las materias económico-financieras se circunscribe básicamente a la etapa de bachillerato. Con el acceso a esta formación de forma libre y gratuita a través de herramientas de aprendizaje electrónico, se facilitaría su acceso desde edades aún más tempranas, incluyendo los niveles de primaria y secundaria, para, de esta manera, facilitar una formación y una cultura financieras básicas que permitieran evitar, en la medida de lo posible, los efectos perniciosos de una nueva 
crisis. De hecho, la evidencia empírica previa demuestra que existe un vínculo directo entre la educación financiera recibida a lo largo de la vida y las decisiones financieras adoptadas individualmente (Cole, Sampson y Zia, 2010), hasta el punto de que la crisis económica de los últimos tiempos ha evidenciado que la falta de educación financiera es uno de los factores que ha contribuido a decisiones financieras erróneas con efectos colaterales muy perjudiciales para la conjunto de la sociedad (OCDE, 2009). Como consecuencia, varios organismos internacionales, como la Organización para la Cooperación y el Desarrollo Económicos (OCDE) y la Comisión Europea, llevan años recomendando que se introduzca la educación financiera en las escuelas desde edades tempranas (OCDE, 2012). La evaluación de esta competencia ha supuesto la materialización del reconocimiento a escala mundial de la importancia de la educación económico-financiera. La preocupación, pues, se ha hecho más evidente en los últimos años, ya que, según parece, cuando la situación económica es favorable pocos se preocupan por gestionar su economía. En cambio, como se extrae de la siguiente cita, cuando empeora, suelen ser más conscientes de la necesidad de tener suficientes conocimientos financieros:

Sí, hubieran hecho falta antes, para no equivocarse a lo mejor en determinadas cosas, a la hora de firmar algunos documentos, pero nunca es tarde para enseñar a los niños, para que no vuelva a suceder... Y referente a los consumidores también para saber qué hacer en determinadas ocasiones (entrevistado 2).

Varias opiniones afirman que la segmentación por edades del módulo es correcta (primaria — de 8 a 10, de 10 a 12 y de 12 a 14-, secundaria y bachillerato). Algunos proponen la publicación de materiales y temas por cursos, aunque enseguida se evidencia que sería excesivo:

Sí, está bien. Ya puestos, la podríamos poner casi por cursos, de tal edad a tal edad, primero de primaria, segundo, tercero..., pero bueno, eso ya... (entrevistado 5).

Sin embargo, algún entrevistado señala que este tipo de herramientas es más adecuado para niños de edades más avanzadas que para niños pequeños, por la capacidad de autogestión de los primeros. Asimismo, destacan la posibilidad de incluir la formación profesional (FP) como un segmento más, al considerar que muchos temas del módulo se estudian en este tipo de formación, en algún ciclo de gestión administrativa: «Yo he echado de menos, quizás, otra segmentación de FP.. porque la FP está orientada a un trabajo como más práctico» (entrevistado 1).

Se contempla la opción de tomar los temas, las actividades y los cuestionarios preparados para bachillerato y adaptarlos para FP. Igualmente, los materiales di- 
rigidos a bachillerato pueden aprovecharse para adultos o ciudadanos en general. Esta adaptabilidad también puede aplicarse a un tema (con sus actividades, cuestionarios, etc.) programado para un nivel inferior, pero interesante para otro superior, incrementando la dificultad. Así, se motiva a los alumnos más avanzados con el reto de acceder a materiales de niveles superiores.

No obstante, se considera que el trabajo realizado en casa con esta herramienta no puede valorarse, al no poderse garantizar su autoría: «No puede comprobarse si lo han utilizado» (entrevistado 2).

Ahora bien, sí puede resultar útil como autoevaluación:

El aspecto autoevaluativo lo veo muy bien. Decir [...], apuntar qué pregunta fallan más, y [...] hacer una estadística en la pizarra. Muy interesante la autoevaluación para que el alumno también se motive y vea si va consiguiendo sus conocimientos (entrevistado 6).

Sin embargo, el módulo no registra las puntuaciones de los cuestionarios:

Puede ser interesante siempre que tengas una herramienta de evaluación, que puedas tener un acceso individualizado del alumno a la aplicación y se quede allí grabado (entrevistado 5).

Se señala la necesidad de comprobar la autoría de las actividades realizadas en línea, por ejemplo, con un examen:

No sabes si lo está haciendo él o quién. Siempre y cuando después haya un examen final, presencial, da igual que el examen presencial sea también hecho a través del ordenador o en papel (entrevistado 5).

Por último, dudan sobre si en las programaciones actuales de las diferentes asignaturas encajan contenidos sobre alfabetización financiera. De no ser así, consideran muy interesante tratarlos, puesto que «son temas que en la vida real a todo el mundo le acaban tocando, ¿no?, y quizás saber un poquito antes de enfrentarte a ello [puede llegar a ser positivo]» (entrevistado 7).

Sin embargo, los entrevistados reconocieron problemas para su implantación por falta de tiempo, ya que a menudo este es limitado:

Cuando los alumnos están más cansados y necesitan un cambio de ritmo, está bien. Ahora, en el día a día, yo creo que no; hay demasiado contenido y poco tiempo para estar dentro de este programa (entrevistado 8).

\subsection{Estructura del módulo}

La funcionalidad y usabilidad son fundamentales en las herramientas de aprendizaje electrónico (Chen, Lin y Kinshuk, 2008). En el caso del módulo analizado, en general, la valoración sobre la estructura es positiva: «Está bien estructurado, 
[... ] es entendible. La facilidad de encontrar las cosas está bastante bien» (entrevistado 4); «fácil de navegar» y «están las cosas categorizadas de diferentes maneras [... , puedes separarlo por materias» (entrevistado 7); «Puedes mirar, si quieres, [...] los exámenes o si quieres, escoger los vídeos» (entrevistado 9); «Muy clara, sí, clara, [... ] de decir aquí debe ser o aquí no [...] [es] bastante clara, sí» (entrevistado 8); «Sí, sí que es fácil de usar [...] y la información está bien presentada, se ve claramente el tema de los enlaces, el tema de lo que estás mirando en cada momento, el tema de volver para atrás [... Sí, es fácil de navegar» (entrevistado 3).

También valoraron positivamente el detalle de cómo aplicar cada tema a una clase, indicando ordenadamente qué actividades deben desarrollarse:

[Es] la guía perfecta para dar una clase, toda la metodología para dar la clase desde principio a fin por un docente, que es muy útil, mucho (entrevistado 1).

Además, en una herramienta en línea es útil incluir enlaces a otras webs interesantes. El módulo de «Alfabetización financiera» contiene, en cada tema, enlaces a otras webs y vídeos relacionados con la temática, que pueden reproducirse en clase o autónomamente en casa: «Te da enlaces a otras páginas donde puedes obtener más [... Está bien [... tanto para el alumno como para el profesor [... que haya un enlace que te lleve a un sitio que puedas [... ] aumentar lo que pone allí. Sí, está bien» (entrevistado 5); «[Los alumnos] están muy acostumbrados a los temas audiovisuales y va bien ilustrar algunas cosas con vídeos» (entrevistado 7).

Asimismo, se considera interesante el acceso restringido de los profesores a algunos apartadosः «Que no se vea cómo hacer la clase [... ] y debería dosificarse la información a los alumnos para que atiendan a las explicaciones del profesor» (entrevistado 4).

\subsection{Contenido del módulo}

La investigación previa, cuando trata de evaluar la idoneidad de herramientas de aprendizaje electrónico, destaca como importante la calidad de los contenidos y de su sistema de actualización (Ozkan y Koseler, 2009). En las entrevistas se destaca que los temas del módulo deberían poder actualizarse con enlaces a noticias interesantes. Así, resultaría aún más interesante para los profesores: «Estaría bien [... ] que te vayan actualizando las cosas y poniendo sobre todo noticias, que tú puedas acceder a ellas, porque desde el punto de vista educativo son buenas» (entrevistado 4).

La valoración de los contenidos forma parte, normalmente, de la evaluación de la satisfacción sobre el funcionamiento de herramientas de aprendizaje elec- 
trónico (Chen y otros, 2011). En este sentido, los entrevistados valoraron positivamente las opciones de actividades y contenidos que se incluyen en el módulo.

Sí, [es útil e interesante para los alumnos] [...] hay contenidos, hay actividades, [... ] actividades interactivas, varias cosas (entrevistado 7).

[...] La actividad de primaria: Si quieres comprar un videojuego y vale 50 euros [...], ¿`cuánto dinero...? [...] Es muy comprensible para los niños para saber el cambio, restar, sumar (entrevistado 8).

Es que lo tienes todo... fichas, vídeos, cuestionarios, todo el trabajo hecho [...]. Te lo tienes que mirar antes de exponerlo, pero ya tienes mucho ganado, y encima, si la fuente es buena, perfecto (entrevistado 9).

Se consideran interesantes algunas lecciones del módulo, como la bolsa, los créditos y los préstamos, los seguros y el dinero, entre otras.

[El tema de la bolsa] lo encuentro muy interesante y se toca poco, [... y considero que tendríamos que saber todos algo de [la] bolsa.

[...] Créditos y préstamos también. La gente no sabe prácticamente ni la diferencia, ni lo que se juega cuando pides un préstamo, porque te atas y dependes luego de devolver mucho dinero que arruina a mucha gente. Es básico conocerlo (entrevistado 1).

Seguros, porque yo misma no estoy demasiado al caso. Y seguro que sí que nos toca a todos de cerca (seguros del coche, del hogar...). Está bien tener una base (entrevistado 7).

Bueno, en uno de los apartados que habla sobre inflación, todos estos contenidos, cosas que oímos y que no las estudiamos, se dicen de forma muy comprensible y están al alcance de todo el mundo. Pienso que son conceptos básicos que todos tenemos que saber (entrevistado 9).

La tarea de evaluación suele ser de interés en la literatura porque, normalmente, es más difícil aplicar formas de evaluación tradicionales a las herramientas de aprendizaje electrónico (Baylari y Montazer, 2009; y Hsu, Chou y Chang, 2011). En cada lección de este módulo de Dolceta, el estudiante puede contestar en línea a dos o más cuestionarios. Si no acierta una respuesta, obtiene un feedback automático. Conocer al instante los aciertos y los errores puede resultar motivador: «Hace bastante atractivo al alumno el “ła ver qué pregunta ahora?, ¿ła ver cuánto acierto?"» (entrevistado 4). Lo que más le ha gustado al entrevistado 4 son los «quizzes, por el hecho de que cuando respondes, tanto si es que sí como si es que no, te están diciendo por qué sí y por qué no. [... [ La explicación está muy bien para los alumnos o el profesor». 
Entre las diferentes actividades o los distintos trabajos propuestos, aunque la mayoría de los entrevistados los consideran adecuados, alguno prefiere las actividades:

Más incluso que los quizzes. Sí, porque un cuestionario lo puede hacer prácticamente todo el mundo y, en ocasiones, pensar en actividades es más complicado y requiere más tiempo (entrevistado 7).

Asimismo, se señala que, aunque hay una lección dedicada a confeccionar presupuestos, «igual podría haber alguna cosa más, por ejemplo, de cómo llevar la economía doméstica. En el sentido de poder ayudar en este caso a las familias» (entrevistado 3).

En conjunto, todo el contenido del módulo es valorado muy positivamente, y se considera básico, completo y suficiente:

Todo está [...]. Necesitas el glosario, después el contenido para desarrollar después actividades, para los vídeos, pienso que es todo el conjunto. Si hiciera falta algo, el docente podría hacerlo teniendo la base. Es más que suficiente (entrevistado 9).

Porque es innovador. No se suele dar. Quizás, en niveles de secundaria y bachillerato y en ciclos formativos, pero estos temas en primaria no se dan y estaría bien que se dieran (entrevistado 5).

Como tienes las actividades, los cuestionarios que te autocorrigen y el temario, [este módulo le] permite que sea muy autodidacta (entrevistado 1).

\subsection{Imagen del módulo}

Alguno de los entrevistados se refirió muy positivamente a la imagen de toda la plataforma de Dolceta:

Lo primero que me ha llamado la atención es el soporte [...] de las imágenes, es muy atractivo, sí. Y [está] muy bien separado. Lo primero que te dice... eliges el país [... ] y luego ya te pone todo el abanico de opciones que tienes (entrevistado 1).

Sí, es atractiva. Sí, no carga, está bien (entrevistado 9).

Centrándonos en el módulo analizado, todos los entrevistados resaltaron su facilidad de uso gracias a su estructura y a su imagen:

Yo lo he visto bastante visual, no sé, hay cuatro grupos. Sí, cuatro lecciones. Te sale el dibujito y también lo relacionas rápido... O sea, lo puedes ordenar por edades, por curso y por ejercicios (entrevistado 2).

Sí, sí, entra bien el tema de los dibujos [...]. El aspecto visual me gusta (entrevistado 3). 
No obstante, los entrevistados no acaban de coincidir en si es lo suficientemente atractiva visualmente para los estudiantes (teniendo en cuenta las edades objetivo). Uno de los entrevistados señaló este punto como un punto que debe mejorarse:

A los niños, a lo mejor no les enganchaba mucho porque es demasiado formal. Para los profesores sí, creo que da [...] confianza y credibilidad por cómo es (entrevistado 2).

Es como... más seria, un poco demasiado compacto para primaria. [En cambio,] las actividades sí que [son] más visuales (entrevistado 7).

Esto contrasta con la opinión de otro entrevistadoः «Con los dibujos se ve más infantil, pero está claro para todos» (entrevistado 7).

Como sugerencia a esta diversidad de opiniones, se propuso que podrían «crearse dos apartados, uno más dedicado a los profesores y el otro a los niños, [... con el mismo contenido» (entrevistado 2).

\subsection{Ventajas e inconvenientes del módulo}

La mayoría solo ven ventajas en este módulo: sencillo de utilizar, fácil de aprender, al alcance de cualquiera y no necesita ninguna tecnología informática específica: «Todo ventajas, un recurso más, solo beneficia» (entrevistado 1); «El tema está bien enfocado, la herramienta está bien montada » (entrevistado 5); «iY tanto!, el estudiante está más motivado si utilizas el ordenador [...]. Bastante intuitiva y rápida de manejar, es fácil... con 10 minutos, porque es todo muy intuitivo, y están muy familiarizados con eso» (entrevistado 6).

Es un recurso concentrado en una única página web, con enlaces de interés a otras webs: «Si realmente tienes ganas de aprender, lo tienes todo en una sola página [... ] muy bien estructurado [...]. Es superútil. Como docente, básicamente agiliza y facilita el trabajo, ¡Y tanto!» (entrevistado 1 ).

Los alumnos pueden utilizarla con o sin ordenadores, porque pueden imprimirse todos los materiales y actividades:

Es una buena herramienta, la fuente es buena. Por ello, todo lo que pone allí lo puedo utilizar algún día. [...] Lo veo bastante fácil y, aparte, como te dan la opción de que no necesitas internet, [de] que lo puedes imprimir, es una buena idea (entrevistado 4).

Sobre todo, por ejemplo, los cuestionarios, porque a veces te convendrá que lo hagan en el ordenador, pero a veces también te convendrá imprimirlo porque te interesará que lo respondan e ir comentándolo (entrevistado 7). 
Contiene actividades que, por ser en línea, son más dinámicas para los alumnos. Es adecuado para completar las clases del profesor:

Sí, [es] divertida. Las actividades eran muy próximas [... ], muy fácil de llevarlas al aula, porque están muy bien explicadas y está todo el trabajo difícil hecho y en determinados temas es difícil pensar en actividades que sean friendly, ¿no? (entrevistado 6).

Es práctico que los estudiantes hagan las actividades en línea: «Algunas de las actividades sí» (entrevistado 7).

Así pues, en conjunto, ven bien el módulo estudiado:

Una herramienta útil y apropiada para acompañar el trabajo del docente, tanto en primaria, secundaria como en bachillerato (entrevistado 6).

Lo ideal sería combinar las dos técnicas [el aprendizaje electrónico y la enseñanza presencial en el aula], porque la explicación teórica durante media hora en una clase es necesaria [...], pero los ejercicios sí que tendrían que ser más interactivos, con el ordenador, y por eso encuentro muy bien la propuesta [... $]$ porque te autocorrige. Es una manera muy práctica de aprender, y muy rápida, [... ] no pierdes tiempo, por ejemplo, en copiar un enunciado (entrevistado 1).

\section{Conclusiones}

Este artículo aporta dos contribuciones relevantes. La primera es la valoración realizada por formadores de una herramienta de aprendizaje electrónico del módulo en línea «Alfabetización financiera» de Dolceta, que ofrece a los profesores de primaria y de secundaria herramientas de formación que pueden beneficiar a la alfabetización financiera básica de sus estudiantes y fomentar una alfabetización financiera de alta calidad, homogénea a escala europea. La segunda contribución es poner de manifiesto la importancia de promover la alfabetización financiera para apoyar las decisiones económico-financieras cotidianas.

Los resultados de la evaluación llevada a cabo muestran el interés y la utilidad de esta experiencia educativa en línea para promover un conocimiento más amplio de los temas financieros cotidianos. Destacan distintos aspectos mejorables para facilitar su utilización en el aula y una mayor adaptación entre diferentes edades y niveles formativos. Entre los aspectos más positivos, se encuentran los siguientes: el contenido ofrecido en Dolceta; su adecuación a las preocupaciones y al entorno financiero actual; las actividades y quizzes incorporados que pueden completar los contenidos teóricos proporcionados en el aula; su estructura y usabilidad; la navegación sencilla por la web; el feedback que proporciona y la posibilidad de que el estudiante aprenda con una mayor autonomía; y la mayor 
motivación que genera en el estudiante no solo por su naturaleza en línea, sino también por su imagen dinámica y atractiva.

El carácter de este estudio, como ya se ha apuntado, es eminentemente descriptivo, ya que su objetivo ha consistido en describir la evaluación de una herramienta en línea específica para fomentar la formación financiera desde edades tempranas. Sin embargo, se considera que a pesar de la especificidad de este trabajo, las lecciones extraídas de él permiten arrojar luz sobre el debate de la utilidad del aprendizaje electrónico, en concreto del aprendizaje aplicado a la mejora de la alfabetización financiera desde edades tempranas. Esto se debe a la conjunción de varias realidades que coexisten y ayudan a explicar la idoneidad de disponer de este tipo de herramientas, y la necesidad, por lo tanto, de evaluar su correcto contenido y funcionamiento, así como de establecer sugerencias que permitan mejorarlas.

Entre estos aspectos de la realidad, destaca, por un lado, la necesidad de la formación financiera (OCDE, 2009 y 2012), que en la actualidad comienza a entenderse como una capacidad clave. Esto se debe a varios motivos, entre los que podrían destacarse: los riesgos financieros que asumen los ciudadanos son cada vez mayores; la longevidad es cada vez mayor; los mercados financieros y crediticios son cada vez más complejos; la necesidad de recursos para afrontar los gastos de salud, jubilación o educación de los hijos ha aumentado; las decisiones de carácter financiero que los ciudadanos adoptan son cada vez más complejas y frecuentes, como consecuencia de los cambios en los mercados y en la economía, incluso aunque se utilicen servicios de asesoría, el responsable último es el ciudadano, que debe entender el servicio de asesoría que se le presta; y los desarrollos tecnológicos han hecho evolucionar la conectividad global y las transacciones financieras, etc. Todos estos cambios aumentan la responsabilidad que asumen los individuos por las decisiones financieras que adoptan. Otro aspecto de la realidad que hay que enfatizar es la necesidad de hacer accesible la formación financiera desde edades tempranas, como plantea la OCDE (2005) en su documento de buenas prácticas para la formación financiera: «dicha educación debería comenzar en la escuela, tan pronto como sea posible». Esto se justifica porque se logra una mayor eficiencia y mejores resultados como consecuencia de dicha alfabetización. Si se facilita pronto, se genera un cambio de cultura y se forma a los individuos para afrontar situaciones económico-financieras complejas que en la actualidad ganan peso a edades cada vez más tempranas. No es raro, pues, que hoy en día los consumidores de servicios y productos financieros puedan ser muy jóvenes y que en su operativa habitual puedan acceder en línea a sus productos financieros y cuentas, efectuar pagos, etc. El último aspecto de la realidad en el 
que hace hincapié este estudio es la idoneidad de disponer de herramientas de aprendizaje electrónico y contenidos en línea para facilitar esta formación. Esto se justifica por la influencia de dichas herramientas formativas en la educación formal, informal y continua de cualquier individuo, y más aún en un contexto en el que desde muy jóvenes el acceso a internet y a las nuevas tecnologías de la información y la comunicación pone a nuestra disposición todo tipo de información, por lo que ejerce una influencia muy importante en nuestra educación. Como señala Aguiar-Perera (2009), «el ordenador y todas sus aplicaciones y funcionalidades se han convertido en instrumentos partícipes de la educación desde edades tempranas» al permitir el desarrollo de distintas capacidades y competencias, y la aplicabilidad de metodologías y herramientas docentes que ayudan a homogeneizar la formación facilitada y a superar las limitaciones temporales y físicas vinculadas a la educación tradicional.

Sin embargo, a pesar de que con este trabajo se pretende justificar la utilidad del aprendizaje electrónico en la mejora de la alfabetización financiera desde edades tempranas a través de la descripción de la valoración de una herramienta concreta, la etapa experimental en la que se encuentra el módulo evaluado no ha permitido acceder a usuarios reales de la herramienta, sino que esta se ha presentado al profesorado involucrado en materias de finanzas, que posteriormente ha realizado su evaluación. Se cree, no obstante, que esta limitación puede constituir una oportunidad, ya que realizar la evaluación antes de la puesta en práctica permite mejorar el módulo de «Alfabetización financiera» antes de su aplicación y contribuye a un mayor conocimiento y utilización de esta herramienta. 


\section{Bibliografía}

Aguiar-Perera, M. V. (2009): «Importancia de trabajar las TIC en educación infantil a través de métodos como la Webquest», Pixel-Bit Revista de Medios y Educación, 34, pp. 81-94.

Alrø, H. y Skovsmose, O. (2000): Student inquiry co-operation in mathematics education, Auckland: Unidad Educativa de Matemáticas (Mathematics Education Unit), Departamento de Matemáticas, Universidad de Auckland.

Arvanitis, S. (2010): «E-learning programs as royalty investments for financial corporations», British Journal of Educational Technology [en línea], 41 (5), pp. 70-74. <http://dx.doi.org/10.1111/j.1467-8535.2009.00971.x>.

Bailey, S. y Kitson, M. (2006): Guidelines for consumer education for adults, CEAN Consumer Education for Adults Network, a Socrates Grundtvig 4 Project 2003-2006.

BARKer, P. (2002): «On being an online tutor», Innovation in education and teaching international [en línea], 39 (1), pp. 3-13. <http://dx.doi. org/10.1080/13558000110097082>.

Baylari, A. y Montazer, G. A. (2009): «Design a personalized e-learning system based on item response theory and artificial neural network approach», Expert System with Applications [en línea], 36, pp. 8013-8021. <http:// dx.doi.org/10.1016/j.eswa.2008.10.080>.

Benito, D. (2009): «Aprendizaje en el entorno del e-learningः estrategias y figura del e-moderador», Revista de Universidad y Sociedad del Conocimiento [en línea], 6 (2), pp. 1-8. <http://dx.doi.org/10.7238/rusc.v6i2.107>.

Burgess, J. (2007)ः «Is a blended learning approach suitable for mature, parttime finance students?», 6th European Conference on E-Learning, pp. 87-95.

Cabero, J. y Gisbert, M. (2005): La formación en internet: guía para el diseño de materiales formativos, Sevilla: MAD.

Carnell, E. y Lodge, C. (2002): Supporting effective learning, Londres: Paul Chapman.

Chen, N.-S.; Lin D.-M., y Kinshuck (2008): «Analysing users' satisfaction with e-learning using a negative critical incidents approach», Innovations in Education and Teaching International [en línea], 45 (2), pp. 115-126. <http:// dx.doi.org/10.1080/14703290801950286>.

Chen, M. L.; Su, Z. Y;; Wu, T. Y;; Shiel, T.-Y., y Chiang, C.-H. (2011); «Influence of dentistry students' e-learning satisfaction: a questionnaire survey», Journal of Medical Systems [en línea], 35(6), pp. 1595-1603.<http:// dx.doi.org/10.1007/s10916-010-9435-x>. 
Cole, S.; Sampson, T., y Zia, B. (2011)ः «Prices or knowledge? What drives demand for financial services in emerging markets?», The Journal of Finance, 66 (6), pp. 1933-1967.

Comisión Europea (2009): The financial crisis and financial education. Report 2nd meeting of the expert group on financial education, Bruselas, pp. 1-6.

Declaración de Bolonia (1999): <http://www.bologna-bergen2005.no/Docs/00Main_doc/990719BOLOGNA_DECLARATION.PDF>.

Fitó-Beltrán, A.; Hernández-Lara, A. B., y Serradell-López, E (2014): "Comparing student competences in a face-to-face and online business game», Computers in Human Behavior [en línea], 30, pp. 452-459. <http:// dx.doi.org/10.1016/j.chb.2013.06.023>.

- (2015): "The effect of competences on learning results an educational experience with a business simulator», Computers in Human Behavior [en línea]. <http://dx.doi.org/10.1016/j.chb.2014.11.003>.

Fu, F. L.; Su, R. C., y Yu, S. C. (2009)ः «EGameFlowः a scale to measure learners' enjoyment of e-learning games», Computers $\mathcal{E}$ Education [en línea], 52 (1), pp. 101-112. <http://dx.doi.org/10.1016/j.compedu.2008.07.004>.

Habschick, M.; Seidl, B., y Evers, J. (2007): Survey of financial literacy schemes in the EU, 27, Hamburgo, pp. 1-108.

Hernández, A. B.; Gorjup, M. T., y Cascón, R. (2010): «The role of the instructor in business games: a comparison of face-to-face and online instruction», International Journal of Training and Development [en línea], 14 (3), pp. 169-179. <http://dx.doi.org/10.1111/j.1468-2419.2010.00350.x>.

Ho, C.y Dzeng, R. (2010)ः «Construction safety training via e-learningः learning effectiveness and user satisfaction», Computers $\mathcal{E}$ Education [en línea], 55, pp. 858-867. <http://dx.doi.org/10.1016/j.compedu.2010.03.017>.

Hsu, J.-L.; Chou, H.-W., y Chang, H.-H. (2011): «EduMiner: using text mining for automatic formative assessment», Expert Systems with Applications [en línea], 38, pp. 3431-3439. <http://dx.doi.org/10.1016/j. eswa.2010.08.129>.

Ma, L*; Vogel, D., y Wagner, C. (2000): «Will virtual education initiatives succeed?», Information Technology and Management [en línea], 1, pp. 209227. <http://dx.doi.org/10.1023/A:1019177109389>.

McGill, T. J. y Klobas, J. E. (2009): «A task-technology fit view of learning management system impact», Computers $E$ Education [en línea], 52, pp. 496508. <http://dx.doi.org/10.1016/j.compedu.2008.10.002>. 
Ministerio de Educación, Cultura y Deporte (2003): Documento marco: la integración del sistema universitario español en el espacio europeo de enseñanza superior, Madrid: Ministerio de Educación, Cultura y Deporte.

Organización para la Cooperación y el Desarrollo EconómiCos (OCDE) (2009): Financial literacy and consumer protection: overlooked aspects of the crisis [en línea]. <http://www.financial-education.org/dataoecd/32/3/43138294.pdf>.

- (2012): PISA 2012 financial literacy assessment framework [en línea]. <http:// www.oecd.org/pisa/pisaproducts/46962580.pdf >.

- (2005): Recommendation on principles and good practices for financial education and awareness, OCDE: Directorate for Financial and Enterprise Affairs.

Ozkan, S. y Koseler, R. (2009): «Multi-dimensional students' evaluation of e-learning systems in the higher education context: an empirical investigation», Computers E Education [en línea], 53 (4), pp. 1285-1296. <http://dx.doi. org/10.1016/j.compedu.2009.06.011>.

Robinson, J. (2008): «Developing a screen-capture reusable learning object for undergraduates», 7th European Conference on E-Learning, 2, Academic Publishing International, pp. 366-375.

Sánchez-Rebull, M. V; Campa-Planas, F. y Hernández-Lara, A. B. (2011): «Dolceta, educación online para los consumidores: módulo de alfabetización financiera en España», El profesional de la información [en línea], 20 (6), pp. 682-688. <http://dx.doi.org/10.3145/epi.2011.nov.13>.

Stoll, L.; Fink, D., y EARL, L. (2004): Sobre el aprender y el tiempo que requiere, Barcelona: Octaedro.

Sun, P. C*; Tsai, R. J*; Finger, G.; Chen, Y. Y*, y Yeh, D. (2008)ः «What drives a successful e-learning? An empirical investigation of the critical factors influencing learner satisfaction», Computers E Education [en línea], 50 (4), pp. 1183-202. <http://dx.doi.org/10.1016/j.compedu.2006.11.007>.

Teo, T., y Wong, S. L. (2013): «Modeling key drivers of e-learning satisfaction among student teachers», Journal of educational computing research [en línea], 48 (1), pp. 71-95. <http://dx.doi.org/10.2190/EC.48.1.d>.

Yamashita, Y*; Takahashi, H., y Terano, T. (2008): «Approach to learning financial theory through business gaming», Proceedings of SICE Annual Conference, 1 (7), pp. 2573-2577. 